\title{
Insights on synergy of materials and structures in biomimetic platelet-matrix composites
}

\author{
Navid Sakhavand ${ }^{1}$ and Rouzbeh Shahsavari ${ }^{1,2,3, a)}$ \\ ${ }^{1}$ Department of Civil and Environmental Engineering, Rice University, 6100 Main Street, Houston, \\ Texas 77005, USA \\ ${ }^{2}$ Department of Material Science and NanoEngineering, Rice University, 6100 Main Street, Houston, \\ Texas 77005, USA \\ ${ }^{3}$ Smalley Institute for Nanoscale Science and Technology, Rice University, 6100 Main Street, Houston, \\ Texas 77005, USA
}

(Received 26 November 2017; accepted 5 January 2018; published online 29 January 2018)

\begin{abstract}
Hybrid materials such as biomimetic platelet-matrix composites are in high demand to confer low weight and multifunctional mechanical properties. This letter reports interfacial-bond regulated assembly of polymers on cement-an archetype model with significant infrastructure applications. We demonstrate a series of $20+$ molecular dynamics studies on decoding and optimizing the complex interfacial interactions including the role and types of various heterogeneous, competing interfacial bonds that are key to adhesion and interfacial strength. Our results show an existence of an optimum overlap length scale $(\sim 15 \mathrm{~nm})$ between polymers and cement crystals, exhibiting the best balance of strength, toughness, stiffness, and ductility for the composite. This finding, combined with the fundamental insights into the nature of interfacial bonds, provides key hypotheses for selection and processing of constituents to deliberate the best synergy in the structure and materials of platelet-matrix composites. Published by AIP Publishing. https://doi.org/10.1063/1.5017200
\end{abstract}

Natural materials such as nacre are primarily biocomposites that are lightweight and exhibit an excellent balance of mechanical properties and multi-functionality far beyond their constituents. ${ }^{1-3}$ In some rare cases, minerals constitute $>95 \%$ of the material's volume as in nacre ${ }^{4}$ or tooth enamel. ${ }^{5}$ With such a high level of minerals, despite being fragile, these materials are tough, durable, and damage tolerant and produce "quasi ductile" behavior. For instance, nacre is made of $95 \%$ aragonite, a brittle mineral, but demonstrates a toughness that is $~ 3000$ times larger than aragonite with the expense of a minor reduction in stiffness. ${ }^{6}$ The quest for understanding and mimicking how nacre, teeth, and other highly mineralized biological materials generate such outstanding mechanical performance despite the weakness of their constituents has long been an engineering pursuit. In this context, one very promising area is engineering bio-inspired synthetic cement-polymer composites for infrastructure applications (e.g., creating earthquake resistance concrete for buildings). This is because the major binding phase of cement hydrate is a layered structure, so-called calcium-silicate-hydrate $(\mathrm{C}-\mathrm{S}-\mathrm{H}),^{7,8}$ a mineral analogous to aragonite that typically shows a brittle behavior. ${ }^{9-12}$ Converting C-S-H to a hybrid polymer/C-S-H composite along with the associated assembly processes, ${ }^{13}$ akin to those of nacre, can potentially confer the needed ductility (or toughness) but is a challenge. This is mainly because of the lack of a fundamental understanding of the interface of the $\mathrm{C}-\mathrm{S}-\mathrm{H}$ and polymers, which has eluded many scientific and engineering efforts to maximize the synergy of parent materials in mechanical performance.

Polymer-cement composites are among matrix-modified cementitious materials which have high potential for

\footnotetext{
a) Author to whom correspondence should be addressed: rouzbeh@rice.edu
}

mechanical property enhancement. ${ }^{14-17}$ Another advantage is that the polymer phase prevents the access of aggressive ions to the micropores of cement, thus enhancing its durability and sustainable performance. ${ }^{18}$ Other applications of polymers in cement include modifying grinding energies of cement crystals. $^{19-22}$ The majority of recent research on polymermodified cementitious composites have been limited to develop more effective techniques to enhance organo-mineral interactions. $^{15,18,23-25}$ This is in part due to a limited understanding of the cement structure and its chemistry and in part because of the complex interaction mechanisms of organicinorganic phases in polymer cementitious materials. ${ }^{26}$ As successful characterizations and experimental probes are becoming available, ${ }^{27,28}$ computational methods can provide key insights into understanding and optimizing the intercalation of the organic phase with the inorganic matter.

Herein, we present a comprehensive MD study on decoding the complex interfacial interactions of polymers and cement with a particular focus on finding the optimum overlap length of the polymers and cement, followed by balancing the mechanical properties such as strength, toughness, stiffness, and ductility. Our model system is a platelet-matrix structure [Figs. 1(a) and 1(b)], which is abundant in nature materials such as nacre where the layered stiff aragonite is arranged in parallel with a soft polymer matrix in between. This layered pattern is a flagship architecture in both natural and biomimetic composites in enhancing mechanical performance. ${ }^{29,30}$ We created a series of polymer-cement composites by putting a soft matrix consisting of polymer strands between tobermorite crystallites, a crystalline analog of C-S$\mathrm{H}$, akin to aragonite [see Fig. 1(a) and Methods in the supplementary material]. Figure 1(c) depicts the snapshot of our simulation system at the initial stage. Two types of common polymer matrices are studied: poly(vinyl) alcohol (PVA) and 

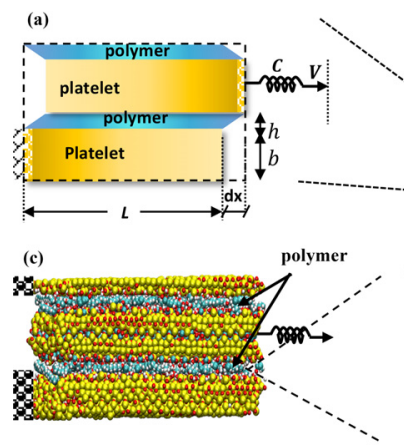

(b)

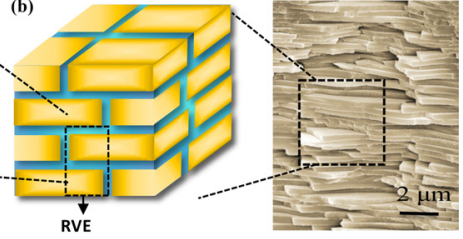

(d)

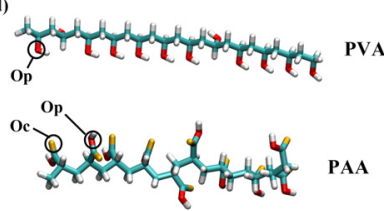

FIG. 1. (a) A representative volume element (RVE) of a platelet-matrix composite and its micrograph (b) (image: Courtesy of Nature Publishing Group). (c) Snapshot of our MD simulation. (d) PVA and PAA as two types of used polymers.

poly(acrylic) acid (PAA) [Fig. 1(d)]. Successful incorporation of both polymer types in $\mathrm{C}-\mathrm{S}-\mathrm{H}$ have been previously reported. ${ }^{15,25}$ PVA has fewer branches than PAA, and it is thus probably easier to bond with tobermorite and form a strong bond. On the other hand, the existence of two different oxygen atoms in PAA (as opposed to the one in PVA) enables PAA to be a donor as well as receiver in hydrogen bond formation.

First, we classify and quantify the heterogeneous $\mathrm{H}$ bond formations and their relative strengths at the polymertobermorite bonding interface. A H-bond forms between a highly electronegative atom as the donor (D) with bounded hydrogen and another polar atom as the acceptor (A). A $\mathrm{H}$-bond is typically defined based on the geometric and/or energetic criteria. A widely used geometric criterion for a $\mathrm{H}$-bond in the triangular HDA bonding formation (Fig. 2) is when $R<3.5 \AA$ and $\beta<30^{\circ}$, where $R$ is the distance between the acceptor and donor atoms and $\beta$ is the angle between D-A and D-H rays. ${ }^{31}$ While this criterion may be appropriate to describe $\mathrm{H}$-bonds in bulk water, we adopt an energetic criterion based on two-dimensional (2D) potential of mean force (PMF) to reduce arbitrariness in defining $\mathrm{H}$ bonds in more complex systems such as polymer-cement. In this criterion, there is a lowest energy basin at short distances for each 2D PMF plot that is constructed based on different choices of angles and distances. This basin corresponds to the H-bonded states and is separated by a saddle point from another higher-energy basin that relates to the non-H-bonded states. Then, the boundary for the H-bond can be delineated by the equipotential contour that passes through the saddle point. Mathematical PMF is quantified as ${ }^{32,33}$

$$
W_{\mathrm{HDA}}(R, \beta)=-k_{B} T \ln \left(g_{\mathrm{HDA}}(R, \beta)\right),
$$

where $T$ is temperature and $k_{B}$ is the Boltzmann constant. $g_{H D A}(R, \beta)$ is the angular-radial distribution function, showing the probability distribution of $R$ and $\beta$ in the formation of HDA. To obtain PMF plots, the polymer chains are initially located on the top of the silica chains with a distance more than a typical $\mathrm{H}$-bond length to prevent initial $\mathrm{H}$-bonding. After molecular dynamics relaxation ${ }^{34,35}$ and investigating PMF plots for various pairs, we found that there are 6 and 8 types of H-bonds in PVA-tobermorite and PAA-tobermorite, respectively, as shown in Figs. 2(a) and 2(b) (to be discussed shortly). This figure illustrates the distribution of $\mathrm{H}$-bonds with varying $R$ and $\beta$ using the PMF plots. The basins and saddle points are obvious in each PMF panel (labeled by white color "B" and "S" letters, respectively). For each panel (e.g., Op-Ow panel), the first atom is the donor and the second atom is the acceptor.

To distinguish the heterogeneity of H-bond strengths in the polymer-tobermorite systems, we calculated the intermittent H-bond Time correlation function (TCF), which is a suitable means to characterize the dynamics of H-bonds. ${ }^{31}$ Using a normalized TCF, the rearrangement time necessary for $\mathrm{H}$-bond formation in a donor-acceptor pair (i.e., $\mathrm{O}_{\mathrm{P}}-\mathrm{O}$ ) is quantified as:
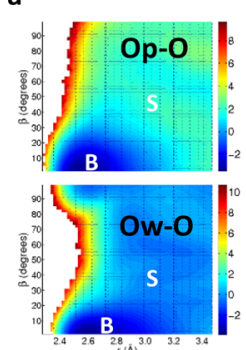

b

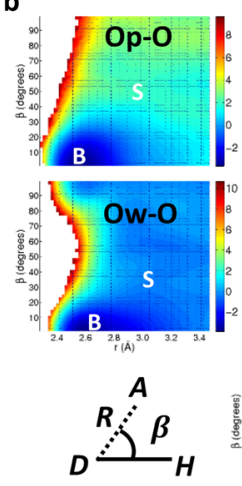

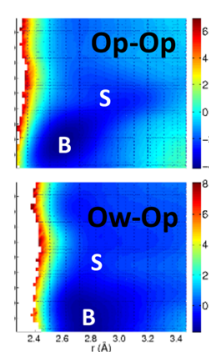
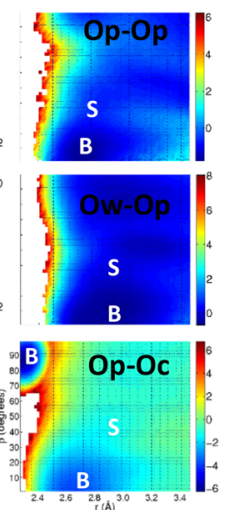
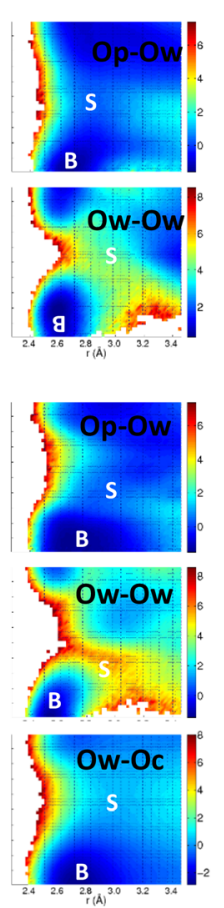

C

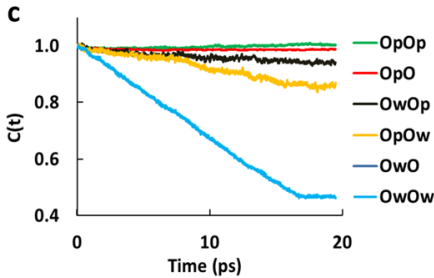

d
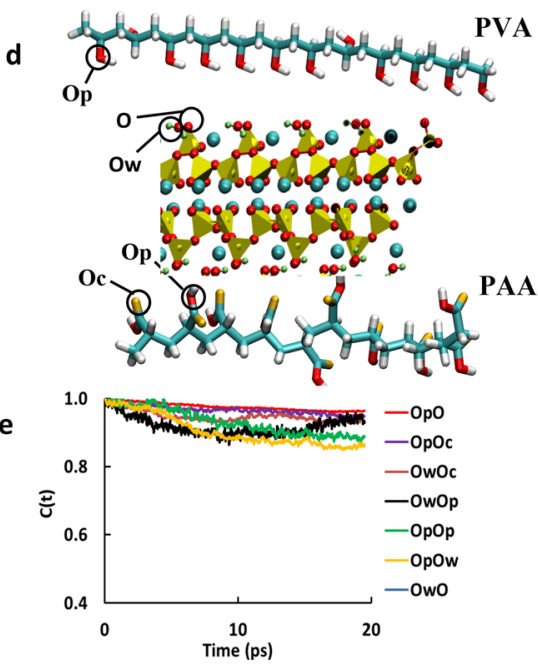

FIG. 2. (a) Potential of mean force plots for $\mathrm{H}$-bonds at the interface of tobermorite-PVA and (b) tobermoritePAA. The values are normalized to $k_{B} T$. The H-bond donor and acceptor are separated by a dashed line, respectively. The symbols "B" and " $\mathrm{S}$ " denote basins and saddle points. (c) Time correlation functions (TCF) for tobermorite-PVA which determines the degree of $\mathrm{H}$-bond formation and breakage as a function of time. (d) Schematic view of PVA (top), tobermorite (middle), and PAA (bottom). Oxygen atoms (donors and acceptors) are shown. Red, yellow, and blue represent $\mathrm{O}, \mathrm{Si}$, and $\mathrm{Ca}$ atoms. (r) TCF for tobermorite-PAA. 


$$
C(t)=\frac{\langle\delta n(t) \delta n(0)\rangle}{\langle\delta n(0) \delta n(0)\rangle}
$$

where, $\delta(t)=n(t)-\langle n\rangle . n(t)$ takes either values of one for H-bonded pairs and zero for not bonded pairs at time $t$. The presence of an $\mathrm{H}$-bond is determined using the $\mathrm{H}$-bond definition explained before. $\langle n\rangle$ is the average value of $n(t)$ over all the pairs and time origins. ${ }^{36} C(t)$ represents the degree of $\mathrm{H}-$ bonds that remained bonded as the system evolves. Starting from a reference time $(t=0), C(t)$ decays with time. Faster decays are representative of weaker H-bonds. Thus, for a perfectly H-bonded pair, $\mathrm{C}(\mathrm{t})$ remains nearly one over time, whereas weak H-bond interactions have deviations from one. In view of H-bond TCF in Figs. 2(c) and 2(d), the relaxation of the Op-O remains very slow even at long time scales, suggesting the high strength of this category of H-bonds. Therefore, we can infer that Op-O H-bonds are the chief source of adhesion between PVA and silicates, acting as the load bearing spots for stress transfer between the organic and inorganic components of this hybrid composite. Analogous conclusions can be made for PAA [Figs. 2(d) and 2(e)].

While the interfacial H-bonding $(\mathrm{Op}-\mathrm{O})$ will be the principal focus of this work, we can rank all the hydrogen bonds from the strongest to the weakest in our systems. In the case of PVA-tobermorite, this ranking is as follows:

1. Op-Op (oxygen of PVA/oxygen of PVA)

2. Op-O (oxygen of PVA/oxygen of silicates)

3. Ow-Op (water/oxygen of PVA)

4. Op-Ow (oxygen of PVA/water)

5. Ow-O (water/oxygen of silicates)

6. Ow-Ow (water/water)

Similarly, the strongest H-bonds in the PAAtobermorite are (in order) as follows:

1. Op-O (oxygen of PAA/oxygen of silicates)

2. Op-Oc (oxygen of PAA/carbon of PAA)

3. Ow-Oc (water/carbon of PAA)

4. Ow-Op (water/oxygen of PAA)

5. Op-Op (oxygen of PAA/oxygen of PAA)

6. Op-Ow (oxygen of PAA/water)

7. Ow-O (water/oxygen of silicates)

8. Ow-Ow (water/water)

In view of the above and Fig. 2, we note the following:

(1) The oxygen atom of the hydroxyl group in PVA (OP) can act as both an acceptor and a donor, likewise the oxygen atom of interlayer water molecules in tobermorite $(\mathrm{OW})$. The oxygen atom of the silica chain in tobermorite is always the acceptor $(\mathrm{O})$. With these atoms, six types of possible $\mathrm{H}$-bond formations are available for the PVA-tobermorite interface.

(2) In addition to the oxygen atom of the hydroxyl group (OP), PAA has another type of oxygen atom which is doublebonded to a carbon atom (OC) and can only be an acceptor; therefore, PAA-tobermorite forms eight types of H-bonds.

(3) Aside from the strong intrinsic intra-molecular H-bonding (Op-Op) in both PVA- and PAA-tobermorite systems, the oxygen atom of polymer and the oxygen of silicates make the strongest interfacial H-bonding.
(4) For stronger H-bonds, it is desirable to have smaller values of $R$ and $\beta$. All the PMF plots show high probability for $\beta<30^{\circ}$ and $R=2.4-3.0 \AA$, confirming the presence of the H-bonds at the polymer-tobermorite interface.

(5) While PVA can only make H-bonds, PAA is charged and can participate in salt bridging (two noncovalent interactions, e.g., hydrogen bonding and electrostatic interactions). This makes the H-bonding network in PAA-tobermorite more complex, as seen by complex PMF plots in Fig. 2(b) and the relatively competing TCF profiles in Fig. 2(e).

(6) Unlike other pairs, $\mathrm{O}_{\mathrm{P}}-\mathrm{O}_{\mathrm{C}}$ in PAA are shown to mostly bond at $90^{\circ}$, arising from covalent-boding of $\mathrm{O}_{\mathrm{P}}$ and $\mathrm{O}_{\mathrm{C}}$ to a carbon atom within individual PAA strands.

Now that the adhesion of the polymer-tobermorite is proved and quantified, we study the mechanical properties associated with these adhesions using 10 different overlap lengths (from $26 \AA$ to $230 \AA$ ) with the goal of finding the optimum overlap length, akin to the nacre and other biological materials which optimize their mechanical properties around a critical overlap length. ${ }^{37,38}$ Our simulation setup resembles a representative volume element of a platelet-matrix structure [Fig. 1(a)]; thus, it is expected that similar results and trends be observed for the large system [Fig. 1(b)]. Figure 3 shows a typical snapshot of a steered molecular dynamics simulation (see Methods in the supplementary material) in which polymer strands slide at the interface while pulling the top tablet. This pulling causes formation and breakage of several $\mathrm{H}-$ bonds, which in turn induces extra ductility.

The presence of multiple stress peaks in Fig. 3 is due to the slip-stick motion, a series of sliding of the polymer strands which results in reduction of stress and large deformation (slip), followed by reformation of H-bonds and increase in stress (stick). This behavior significantly increases toughness of the system. Note that in this paper, we use the energy definition of the "toughness," which is described as the amount of the energy per volume a material absorbs before it fails, i.e., area under the stress-strain curve. ${ }^{34,39-43,46}$ Although related, this definition is different than the classical definition of fracture toughness with the unit of $\mathrm{Pam}^{0.5}$. Within the multiple peaks in force-displacement plots, the first stress peak is typically larger. As seen in the snapshots of Fig. 3, while the middle tobermorite is pulled along, the opportunity for H-bonding decreases because fewer polymers and less interface area are available. The first stress peak, therefore, represents the strength of the system. Other mechanical properties are also derived from the stress-strain plots. Stiffness is defined as the slope of the first stick stage. The strain is calculated by

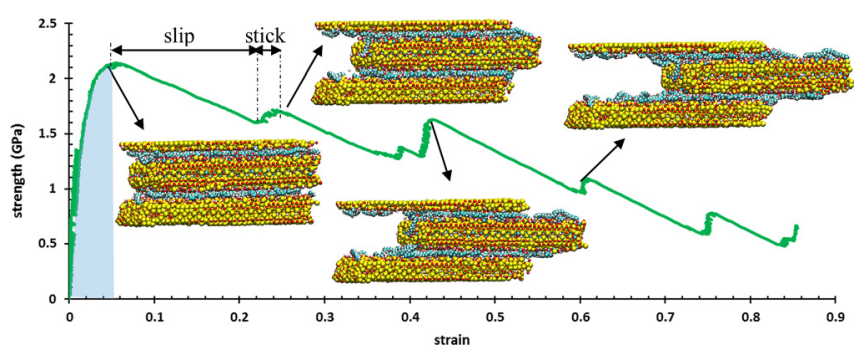

FIG. 3. A typical stress-strain plot of the steered MD simulation. 
dividing the horizontal displacement $[d x$ in Fig. 1(a)] of the pulled atoms by the overlap length $(L)$. While the ultimate ductility and toughness are higher due to the slip-stick motion, for consistency with the above representation, we consider strain and elastic energy density when the first stress peak is achieved. The analytical expressions for the toughness $\left(W_{\text {total }}\right)$ of a unit cell of an archetype platelet-matrix composites (with dissimilar platelets) is ${ }^{29,33}$

$$
\begin{aligned}
W_{\text {total }}= & \frac{1}{L\left(b_{1}+b_{2}+h\right)}\left[h \int_{0}^{L} \frac{\tau(x)^{2}}{2 G} d x\right. \\
& \left.+b_{1} \int_{0}^{L} \frac{\sigma_{1}(x)^{2}}{2 E_{1}} d x+b_{2} \int_{0}^{L} \frac{\sigma_{2}(x)^{2}}{2 E_{2}} d x\right],
\end{aligned}
$$

where $G$ is the shear modulus of the polymer, $\tau(x)$ is the shear stress across the overlap length $L, \sigma_{1}(x)$ and $\sigma_{2}(x)$ are the axial stresses within the top and bottom tobermorite, $b_{1}$ and $b_{2}$ are the thicknesses of the top and bottom tobermorite platelets, and $E_{1}$ and $E_{2}$ are the elastic moduli of the top and bottom tobermorite platelets. Obviously, in our case, the platelets are identical; thus, $\sigma_{1}(x)=\sigma_{2}(x)$ and $b_{1}=b_{2}$ and $E_{1}=E_{2}$

To study the influence of the overlap length on the complex H-bonding and thus the mechanical properties of the composites, we kept the thickness of the tobermorite $(b)$ constant while varying the overlap length $L$ by creating tobermorite supercells with varying unit lengths. In Eq. (3), toughness is defined as the elastic energy density, which is composed of two parts: shear strain energy associated with the shear deformations of the matrix (polymer) and the axial strain energy associated with the axial deformations of the platelets (tobermorite). The sum is the toughness, which corresponds to the area under the stress-strain curve until the first H-bond rupture (shaded area in Fig. 3).

Figure 4 clearly shows that strength and stiffness increase with the overlap length, $L$, and eventually saturate, albeit with different rates. Increasing both ductility and strength helps increasing toughness (the area under the stress-strain plot). The inverse relationship of ductility and strength versus $L$ suggests that toughness is maximized at a finite length. This is confirmed with the results shown Fig. 4, in line with the theoretical prediction for such optimized toughness at critical length scales of platelet-matrix composites. ${ }^{29}$ In view of Fig. 4 and given that each mechanical property behaves differently with increasing overlap length, $L$, the optimum overlap length, $L^{o p t}$, to balance the mechanical properties in the given architecture of tobermorite-PVA would be $L \sim 120 \AA$ and for tobermorite-PAA is $L \sim 150 \AA$. These length scales suggest that PVA/PAA-cement composites would exhibit their best combination of strength, toughness, stiffness, and ductility when the cement mineral has a finite lateral size (here $\sim 12-15 \mathrm{~nm}$ ). This optimum length scale ensures that there is no significant compromise in inversely related properties such as strength and toughness. In other words, values more than $15 \mathrm{~nm}$ may marginally increase strength, but there will be a significant drop in ductility and/or toughness [c.f. Figures 4(a) and 4(c)]. Similarly, values that are much smaller than the optimum length will lead to a sacrifice in both strength and toughness since there is no sufficient overlap for stress transfer. Around the
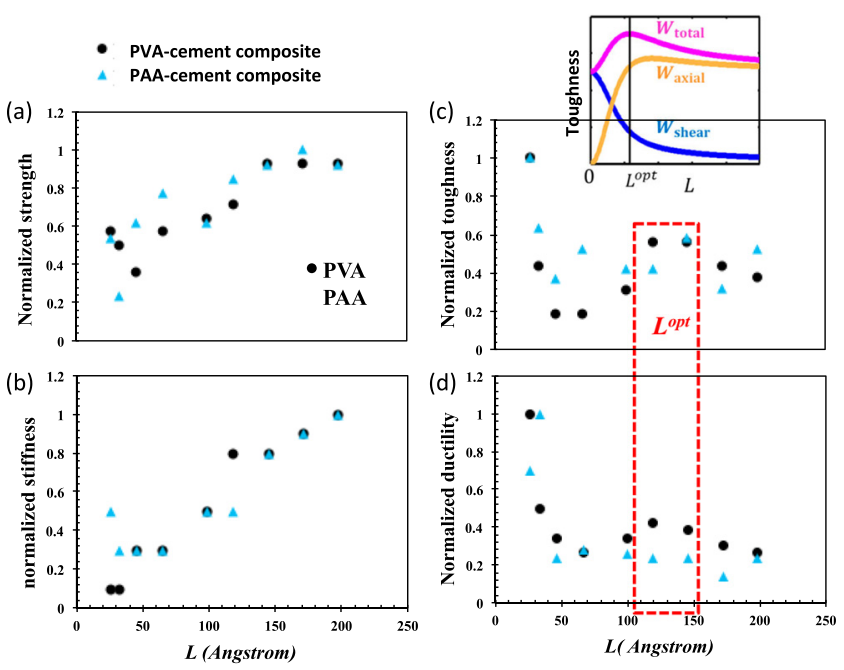

FIG. 4. Strength (a), stiffness (b), toughness (c) and ductility (d) vs overlap length.

optimum length, not only toughness is optimized but both strength and stiffness exhibit highly satisfactory values ( $\sim 80 \%-90 \%$ of their saturation values). This combination of properties represents an excellent balance in mechanical properties, an attribute that is key to the mechanics of several natural platelet-matrix composites. The underlying physical reason behind the existence of this optimum overlap length lies in the opposite behavior of the shear and axial strain energies of the system versus $\lambda L$, as shown in the inset of Fig. 4(c). Theoretical studies ${ }^{29,33}$ have shown that the shear strain energy associated with the polymer deformation decreases as the overlap length increases while the axial strain energy associated with the platelets increases and then saturates. Given that the toughness is the sum of the shear and axial strain energies, the aforesaid opposite trends lead to an optimum spot (finite $\lambda L$ ), hence maximizing the toughness. This fundamental insight can assist experimentalists to optimize their composite design. For instance, one can tune the synthesis conditions and processing routes to yield cement building blocks with a desired platelet length and/or select appropriate polymer types with right molecular weight. In fact, our group recently synthesized de novo cement building blocks with various shapes and sizes, ${ }^{44}$ paving the path towards the bottom-up design and assembly of cement-matrix composites. Such a strategy in composite design would guarantee the highest chance of synergy in the merger of the structure and materials in conferring multifunctionality in synthetic platelet-matrix composites.

In summary, this study provided new fundamental insights into decoding and optimizing the key interfacial interactions in biomimetic platelet-matrix composites. By modeling two common polymers (PVA and PAA) on a typical cement model, we provided a deep understanding of the heterogeneity, strength, and competing mechanisms of forming hydrogen bonds in each system and rank their strengths. In both systems, aside from the strong intrinsic intramolecular H-bonding within the polymer (Op-Op), the oxygen atom of polymer and the oxygen of silicates make the strongest H-bonding, governing the interfacial adhesion of the organic and inorganic phases. While PVA can only make 
H-bonds, PAA is charged and can participate in salt bridging, making the H-bonding network more complex. By closely monitoring the synergy of the structure and materials for various overlap lengths, we identified the optimum overlap length for the chosen architectures to be $L \sim 120 \AA$ for the PVA-cement and $L \sim 150 \AA$ for the PAA-cement model. Such optimal lengths enable balancing mechanical properties of polymer/cement composites. Broadly, this work can have implications for other damage-tolerant composites made of hard and soft building blocks but arranged in different architectures. $^{2,5,45}$

\section{See supplementary material for detailed methods.}

We acknowledge support from National Science Foundation (NSF) Grant No. CMMI-1538312.

${ }^{1}$ W. J. Clegg, K. Kendall, N. M. Alford, T. W. Button, and J. D. Birchall, Nature 347(6292), 455-457 (1990).

${ }^{2}$ M. A. Meyers, J. McKittrick, and P.-Y. Chen, Science 339(6121), 773-779 (2013).

${ }^{3}$ H. Peterlik, P. Roschger, K. Klaushofer, and P. Fratzl, Nat. Mater. 5(1), 52-55 (2006).

${ }^{4}$ J. D. Currey and J. D. Taylor, J. Zool. 173(3), 395-406 (1974).

${ }^{5}$ M. Yahyazadehfar, D. Bajaj, and D. D. Arola, Acta Biomater. 9(1), 4806-4814 (2013).

${ }^{6} \mathrm{M}$. Sarikaya and I. Aksay, Biomimetics: Design and Processing of Materials, AIP Series in Polymers and Complex Materials (AIP, Woodbury, NY, 1995).

${ }^{7}$ R. Shahsavari, Hierarchical Modeling of Structure and Mechanics of Cement Hydrate (MIT Press, 2011).

${ }^{8}$ R. Shahsavari, M. J. Buehler, R. J. M. Pellenq, and F. J. Ulm, "First-principles study of elastic constants and interlayer interactions of complex hydrated oxides: Case study of tobermorite and jennite," J. Am. Ceram. Soc. 92(10), 2323-2330 (2009).

${ }^{9}$ N. Zhang and R. Shahsavari, "Balancing strength and toughness of calcium-silicate-hydrate via random nanovoids and particle inclusions: Atomistic modeling and statistical analysis," J. Mech. Phys. Solids 96, 204-222 (2016).

${ }^{10}$ N. Zhang, P. Carrez, and R. Shahsavari, "Screw-dislocation-induced strengthening-toughening mechanisms in complex layered materials: The case study of tobermorite," ACS Appl. Mater. Interfaces 9(2), 1496-1506 (2017).

${ }^{11}$ L. Tao and R. Shahsavari, "Diffusive, displacive deformations and local phase transformation govern the mechanics of layered crystals: The case study of tobermorite," Sci. Rep. 7(1), 5907 (2017).

${ }^{12} \mathrm{~S}$. Jalilvand and R. Shahsavari, "Molecular mechanistic origin of nanoscale contact, friction, and scratch in complex particulate systems," ACS Appl. Mater. Interfaces 7(5), 3362-3372 (2015).

${ }^{13}$ A. Picker, L. Nicoleau, Z. Burghard, J. Bill, I. Zlotnikov, C. Labbez, A. Nonat, and H. Cölfen, Sci. Adv. 3(11), e1701216 (2017).

${ }^{14}$ A. Popova, G. Geoffroy, M.-F. Renou-Gonnord, P. Faucon, and E. Gartner, J. Am. Ceram. Soc. 83(10), 2556-2560 (2000).

${ }^{15}$ S. C. Mojumdar and L. Raki, in Proceedings of the American Ceramic Society 107th Annual Meeting (Wiley, 2005), pp 1-10.

${ }^{16}$ R. Shahsavari and N. Sakhavand, "Hybrid cementitious materials: Nanoscale modeling and characterizations," in Innovative Development of Advanced Multifunctional Nanocomposites in Civil and Environmental Engineering (Woodland Publishing, 2016).

${ }^{17}$ J. J. Biernacki, J. W. Bullard, G. Sant, K. Brown, F. P. Glasser, S. Jones, T. Ley, R. Livingston, L. Nicoleau, J. Olek, F. Sanchez, R. Shahsavari, P. E. Stutzman, K. Sobolev, and T. Prater, "Cements in the 21st century: Challenges, perspectives, and opportunities," J. Am. Ceram. Soc. 100(7), 2746-2773 (2017).

${ }^{18}$ J. J. Beaudoin, L. Raki, and R. Alizadeh, "A 29Si MAS NMR study of modified C-S-H nanostructures," Cem. Concr. Compos. 31(8), 585-590 (2009).

${ }^{19}$ R. Shahsavari, L. Chen, and L. Tao, "Edge dislocations in dicalcium silicates: Experimental observations and atomistic analysis," Cem. Concr. Res. 90, 80-88 (2016).
${ }^{20} \mathrm{R}$. Shahsavari, L. Tao, and L. Chen, "Structure, energetics, and impact of screw dislocations in tricalcium silicates," J. Am. Ceram. Soc. 99(7), 2512-2520 (2016).

${ }^{21}$ R. Shahsavari and L. Chen, "Screw dislocations in complex, low symmetry oxides: core structures, energetics, and impact on crystal growth," ACS Appl. Mater. Interfaces 7(4), 2223-2234 (2015).

${ }^{22}$ Mishra, R. K. Mohamed, A. K. Geissbühler, D. Manzano, H. Jamil, T. Shahsavari, R. Kalinichev, A. G. Galmarini, S. Tao, L. Heinz, H. Pellenq, R. van Duin, A. C. T. Parker, S. C. Flatt, R. J. Bowen, and P. cemff, "A force field database for cementitious materials including validations, applications and opportunities," Cem. Concr. Res. 102(Supplement C), 68-89 (2017).

${ }^{23}$ F. Merlin, H. Lombois, S. Joly, N. Lequeux, J.-L. Halary, and H. Van Damme, J. Mater. Chem. 12(11), 3308-3315 (2002).

${ }^{24}$ R. Alizadeh, J. Beaudoin, L. Raki, and V. Terskikh, J. Mater. Sci. 46(2), 460-467 (2011).

${ }^{25}$ S. C. Mojumdar and L. Raki, J. Therm. Anal. Calorim. 86(3), 651-657 (2006).

${ }^{26}$ L. Raki, J. Beaudoin, R. Alizadeh, J. Makar, and T. Sato, Materials 3(2), 918-942 (2010).

${ }^{27}$ R. A. Perez, H.-W. Kim, and M.-P. Ginebra, J. Tissue Eng. 3, 2041731412439555 (2012).

${ }^{28}$ J. Minet, S. Abramson, B. Bresson, C. Sanchez, V. Montouillout, and N. Lequeux, Chem. Mater. 16(20), 3955-3962 (2004).

${ }^{29}$ N. Sakhavand and R. Shahsavari, "Universal composition-structure-property maps for natural and biomimetic platelet-matrix composites and stacked heterostructures," Nat. Commun. 6, 6523 (2015).

${ }^{30}$ S. Farzanian and R. Shahsavari, "Mapping the coupled role of structure and materials in mechanics of platelet-matrix composites," J. Mech. Phys. Solids 112, 169-186 (2018).

${ }^{31}$ A. Luzar and D. Chandler, Nature 379(6560), 55-57 (1996).

${ }^{32}$ R. Kumar, J. R. Schmidt, and J. L. Skinner, J. Chem. Phys. 126(20), 204107 (2007).

${ }^{33}$ N. Sakhavand, P. Muthuramalingam, and R. Shahsavari, "Toughness governs the rupture of the interfacial H-bond assemblies at a critical length scale in hybrid materials," Langmuir 29(25), 8154-8163 (2013).

${ }^{34}$ N. Sakhavand and R. Shahsavari, "Dimensional crossover of thermal transport in hybrid boron nitride nanostructures," ACS Appl. Mater. Interfaces 7(33), 18312-18319 (2015).

${ }^{35}$ F. Shayeganfar and R. Shahsavari, "Oxygen- and lithium-doped hybrid boron-nitride/carbon networks for hydrogen storage," Langmuir 32(50), 13313-13321 (2016).

${ }^{36}$ J. M. Haile, Molecular Dynamics Simulation (John Wiley \& Sons, Inc., New York, 1992).

${ }^{37}$ H. D. Espinosa, J. E. Rim, F. Barthelat, and M. J. Buehler, Prog. Mater. Sci. 54(8), 1059-1100 (2009).

${ }^{38}$ X. Wei, M. Naraghi, and H. D. Espinosa, ACS Nano 6(3), 2333-2344 (2012).

${ }^{39}$ N. D. Kim, A. Metzger, V. Hejazi, Y. Li, A. Kovalchuk, S.-K. Lee, R. Ye, J. A. Mann, C. Kittrell, R. Shahsavari, and J. M. Tour, ACS Appl. Mater. Interfaces 8(20), 12985-12991 (2016).

${ }^{40}$ N. Sakhavand and R. Shahsavari, "Synergistic behavior of tubes, junctions, and sheets imparts mechano-mutable functionality in 3D porous boron nitride nanostructures," J. Phys. Chem. C 118(39), 22730-22738 (2014).

${ }^{41}$ R. Shahsavari and N. Sakhavand, "Junction configuration-induced mechanisms govern elastic and inelastic deformations in hybrid carbon nanomaterials," Carbon 95, 699-709 (2015).

${ }^{42}$ S. H. Hwang and R. Shahsavari, "Intrinsic size effect in scaffolded porous calcium silicate particles and mechanical behavior of their self-assembled ensembles," ACS Appl. Mater. Interfaces 10, 890-899 (2018).

${ }^{43}$ M. A. Rafiee, T. N. Narayanan, D. P. Hashim, N. Sakhavand, R. Shahsavari, R. Vajtai, and P. M. Ajayan, "Hexagonal boron nitride and graphite oxide reinforced multifunctional porous cement composites," Adv. Funct. Mater. 23(45), 5624-5630 (2013).

${ }^{44}$ S. E. Moghaddam, V. Hejazi, S. H. Hwang, S. Sreenivasan, J. Miller, B. Shi, S. Zhao, I. Rusakova, A. R. Alizadeh, K. H. Whitmire, and R. Shahsavari, "Morphogenesis of cement hydrate," J. Mater. Chem. A 5(8), 3798-3811 (2017).

${ }^{45}$ S. H. Hwang, J. B. Miller, and R. Shahsavari, "Biomimetic, strong, tough, and self-healing composites using universal sealant-loaded, porous building blocks," ACS Appl. Mater. Interfaces 9(42), 37055-37063 (2017).

${ }^{46} \mathrm{R}$. Shahsavari, "Intercalated hexagonal boron nitride/silicates as bi-layer multifunctional ceramic,” ACS Appl. Mater. Interfaces 10, 2203-2209 (2018). 